Folgerungen daraus ziehen; als Ausnahme möchte ich auf eine unter der Leitung yon A. Gockel kürzlich von J. Wojtaszewski ausgeführte Arbeit hinweisen ${ }^{24}$ ), in der gezeigt wird, daß die Neigung von Niederschlägen, bei ihrer Bildung aus Lösungen Uran $X$ mitzureißen, ganz parallel geht mit ihrer Fähigkeit, Uran X zu adsorbieren, wenn sie auberhalb der Lösung hergestellt und dann in ihr geschüttelt werden. Ich habe mich durch Versuche überzeugt, daB diese Regel auch bei andern Radioelementen zutrifft; und da, besonders nach einer unlāngst veröffentlichten Arbeit von $K$. Fajans und P. Beer ${ }^{25}$, die Bedeutung der chemischen Natur der Niederschläge für das $M$ i tr e i $b$ e $n$ der

24) J. Wojtaszewski, Ueber das Ausfallen einiger radioaktiver Stoffe aus wässerigen Lösungen (Freiburg 1913).

25) K. Fajans und P. Beer, Ber. d. Deutsch chen. Ges. 46, 3486 (1913).
Radioelemente feststeht, würde eine Bestätigung der Regel an einem größeren experimentellen Material mit Notwendigkeit dazu führen, di e s e Adsorptionen nicht als rein physi$k a l i s c h e n$ Vorgang a ufufassen, sondern in nahe Beziehung zu den Vorstellungen äber chemische Affinität $z u$ bringe ${ }^{26}$ ). Ueber die in dieser Richtung angestellte Untersuchung wird in einiger Zeit berichtet werden.

20) DaB z. B., wie ich beobachtet habe, aus einer Lossung von Thorium $\mathrm{B}$ und Thorium $\mathrm{C}$ durch $\mathrm{BaSO}_{4}$ mehr Th $\mathrm{B}$, durch $\mathrm{CuO}$ mehr $\mathrm{Th} C$ adsorbiert wird, $1 \mathrm{aBt}$ sich auf Grund der Ansicht von Fre und $11 \mathrm{ch}$ nicht erklären, zeigt aber (Thorium $B=B$ Bei, Thorium $C=$ Wismut) einen deutichen Zusammenhang mit dem chemischen Vethalten. Ob die andern für Adsorption charakteristischen GesetzmäBigkeiten hier gelten und ob man demnach berechtigt ist, auch diese Erscheinungen mit dem gemeinsamen Namen "Adsorption" zu bezeichrien, wird die nähere Untersuchung lehren.

\title{
Ueber die Zusammensetzung des Lackes, welcher auf dem baumwollenen Gewebe bei der Färbung mit basischen Farbstoffen gebildet wird.
}

\author{
Von A. Sanin (Kiew).
}

Die basischen Farbstoffe können, wie bekannt, auf dem baumwollenen Gewebe nicht unmittelbar fixiert werden; zur Fixierung dieser Stoffe muß man das Gewebe zuerst mit der Tanninlösung imprägnieren, darauf es mit der Brechweinsteinlösung oder einem anderen Antimonpräparat bearbeiten und nach diesen Operationen mit diesem oder jenem Farbstoff färben. Es werden also auf das Gewebe drei Substanzen aufgetragen, welche in eine bekannte Reaktion zwischen einander eintretend eine beständige Verbindung gegen verschiedene chemische Reagenzien bilden.

Die erwähnte Fixierungsmethodederbasischen Farbstoffe auf der Baumwolle wurde vor fast 50 Jahren vorgeschlagen, aber trotzdem bleibt das Wesen des chemischen Prozesses der Färbungsmethode mit diesen Farbstoffen bisher noch unbekannt. In der technischen Literatur wurde die Frage: „Was für eine Verbindung sich zwischen dem Tannin, Brechweinstein und dem Farbstoffe auf der Baumwolle bildet? " fast gar keiner Betrachtung unterzogen. Ganswindt spricht in seinem Buche "Die Gerbstoffe als Beizen" die Meinung aus, dab das Wesen der Lackbildung auf der zwischen dem Tannin von Antimontannat und den Farbstoffen sich vollziehenden Wechselwirkung beruht. Diese Erklärung der Lackbildung wurde vor ca. 20 Jahren ausgesprochen und bleibt, wie es scheint, bisher noch die einzige.

Für Lackbildung sind also notwendig drei Substanzen: das Tannin, der Brechweinstein und der basische Farbstoff. Wenn man diese drei Substanzen gleichzeitig zur Reaktion zu bringen und die Zusammensetzung der erhaltenen Niederschläge $z u$ untersucher unternimmt, so erweist sich, dab sie nicht beständig bleibt. Die Zahlen der Analyse schwanken in ziemlich breiten Grenzen in Abhängigkeit von verschiedenen Bedingungen ${ }^{1}$ ). Es ist wahrscheinlich, dab es. sich hier um die Bildung von Adsorptionsverbindungen handelt. Die Ursache solcher Erscheinungen darf man in der Fähigkeit des. kolloiden Tannins suchen, sich von den erhaltenen Niederschlägen adsorbieren zu lassen und wohl auch selbständige chemische oder Adsorptionsverbindungen mit dem Brechweinstein oder den Farbstoffen einzugehen.

Ich habe schon in früheren Arbeiten ${ }^{2}$ ) darauf hingewiesen, daß das Tannin und basische Farbstoffe beständige chemische Verbindungen bilden, wenn in der Losung ein Ueberschuß von Farbst of vorhanden ist, und Adsorptions-

1) Diese Zahlen werden in einer besondaren Abhandlung mitgeteilt werden.

2) Zeitschr. f. Farb.-Ind., Heft 6, 7 (1911 
verbindungen, wenn das Tan nin im Ueberschuf ist. Dasselbe findet bei der gegenseitigen Reaktion des Tannins und des Brechweinsteins statt. Auferdern wurde von mir bemerkt, daB dor Brechweinstein mit basischen Farbstoffen auch verschiedene Verbindungen gibt.

Es ergibt sich aus dem Angeführten, daß es für das Erbalten von beständigen chemischen Verbindungen notwendig ist, mancheBedingu ugen za erfüllen, insbesondere die Substanzen in die Reaktion allmählich einzuführen und den Ueberschul von Tannin in der Lösung zu vermeiden.

Der Prozeb der Lackbildung auf dem Gewebe in der Technik befriedigt gerade die oben angegebenen Bedingungen. Man imprägniert in der Tat ças Gewebe zuerst mit Tanninlosung, darauf mit überschüssiger Brechweinsteinlosung, nachher wäscht man sorgfältig das Gewebe, um den in Reaktion nicht eingetretenen Brechweinstein zu entfernen. Nach meinen Untersuchungen bildet sich bei diesen Bedingungen (Abwesenheit des Tanninüberschusses, Reaktion in der Kälte, verdünnte Lösungen) eine beständige chemische Verbindung der Zusammensetzung $\left\{\mathrm{C}_{14} \mathrm{H}_{9} \mathrm{O}_{4}\right)_{2} \mathrm{SbOH}$, welche man als einen $\mathrm{Ab}$ kömmling der antimonigen Säure betrachten kann. Bei der weiteren Bearbeitung des Gewebes mit basischen Farbstoffen tritt das auf dem Gewebe aufgetragene antimongerbsaure Salz mit dem Farbstoffe in eine neue Verbindung ein.

Wenn man die Bildung von chemischen Verbindungen vermutet, so ergibt sich die Frage: Auf welche Weise findet die Vereinigung der Farbstoffe mit dem antimongerbsauren Salz statt? Ganswind $t$ behauptet, daß der Farbstoff mit dem Tannin des gerbsauren Salzes in eine Verbindung eintrete. Nach meiner Meinung kann die Farbstoffbase mit dem Tannin des Antimontannats sich nicht vereinigen, da die Säureeigenschaften des Tannins durch das Antimon gesättigt werden. Es ist richtiger nach meiner Ansicht, die Vermutung zu machen, dah die Farbstoffbase die Säureeigenschaften des Hydroxyls der antimonigen Säure sättigt, d. h., dab sich eine Verbindung der antimonigen Säure, in welcher zwe! Hydroxyle durch die Tanninreste und eines durch die Farbstoffbase ersetzt wird, bildet. Bei solcher Vermutung ist es unurrigänglich notwendig, das Freiwerden der Mineralsäure des Earbstoffes anzunehnen. In der. Tat gelang es mir, in dern nach der Färbung zurückgebliebenen und ganz erschöpften Bade die Salzsäure zu entdecken; der im Glas aber vorbereitete und gut abgewaschene Lack enthielt kein Chlor.
Diese vorlätriggen Erfahrungen führen also zu dem Schlusse, dab das antimongerbsaure 3alz in eine chemische Reaktion mit dem Farbstoffe eintritt, und dap diese Vereinigung in der beim Antimon stehender OH-Gruppe vorgeht. Auf die letzte Schlubfolgerung weist auch folgender Versuch hin: Wenn das mit Tannin imprägnierte Gewebe in eine sehr starke Mischlosung des Brechweinsteins und des Kochsalzes eingelaucht wird, so wird das so vorbereitete Gewebe sehr wenig mit Farbstoffen gefärbt. Der Grund dieser Erscheinung ist der, dafs sich bei der Tanninfixierung mit starker Brechweinstein- und Kochsalzlösung nicht das Salz $\left.\mathrm{C}_{14} \mathrm{H}_{9} \mathrm{O}_{9}\right)_{2} \mathrm{SbOH}$, sondem ein Salz $\mathrm{C}_{14} \mathrm{H}_{9}(\mathrm{SbO}) \mathrm{O}_{9}$ bildet, was die Möglichkeit einer Reaktion dieses Salzes mit dem Farbstoffe ausschliefit. Der angestellte. Versuch hat dies vollkommen bestätigt. Zwei baumwollene mit Tannin imprägnierte Streifen wurden

1. in der Brechweinsteinlösung $7,5 \mathrm{~g}$ pro Liter und

2. in der Brechweinsteinlösung $200 \mathrm{~g}$ $+300 \mathrm{~g} \mathrm{NaCl}$ pro Liter

fixiert und in Kristallviolettlösung gefärbt. Die bei $100^{\circ} \mathrm{C}$ getrockneten Streifen wurden vor and nach der Färbung gewogen, um die befestigte Farbstoffnenge zu erfahren. Es erwies sich auf dem ersten Streifen $0,0232 \mathrm{~g}$, auf dem zweiten nur $0,0091 \mathrm{~g}$ des Farbstoffes, obgleich auf dam ersten $0,0479 \mathrm{~g}$ das antimongerbsauren Salzes $\left(\mathrm{C}_{14} \mathrm{H}_{9} \mathrm{O}_{9}\right)_{2} \mathrm{SbOH}$, aber auf dem zweiten $0,0608 \mathrm{~g}$ des Salzes $\mathrm{C}_{14} \mathrm{H}_{9}(\mathrm{SbO}) \mathrm{O}_{9}$ vor handen war. Die geringe Färbung des Gewebes des zweiten Streifens kann man nur durch die Adsorptionserscheinungen erklären.

Um die Wechselwirkung $z$ wischen dem Tannin, Brechweinstein und dem Farbstoffe, in welche diese Substanzen während der Reaktion einireten, annäherungsweise festzustellen, machte ich folgende Versuche: Ich bereitete drei äquivalente Lösungen von Tanniu, Brechweinstein und Kristallviolett, goB in mehrere Giäser $90 \mathrm{ccm}$ ron der Tanninlösung, fügte sodann in jedes Gas $10 \mathrm{ccm}$ von der Brechweinsteinlösung und eine kleine Menge von der Natriumazetatlösung hinzu; bei diesen Bedingungen bildete sich das $\mathrm{Salz}\left(\mathrm{C}_{14} \mathrm{H}_{9} \mathrm{O}_{9}\right)_{2} \mathrm{SbOH}$, welches beim Zusetzen der Natriumazetatlossung in den Niederschlag uberging. Zu den bis za $70-80^{\circ} \mathrm{C}$ erhitzten l.ösungen wurden in das erste Glas $8 \mathrm{ccm}$ von der Kristallviolettlösung, in das zweite $9 \mathrm{~cm}$, in das dritte $10 \mathrm{ccm}$, in das vierte $11 \mathrm{ccm}$ hinzugefügt. Die Lösungen werden darauf abgekühlt und in 
gleiche Probiergläser filtriert. Nach der Farbe des Filtrats urteilte ich über das Reaktionsende. Die Beobachtung zeigte, daß das Optimum $10 \mathrm{ccm}$ der Farbstofflosung sind, welche gerade eincm Molekül des Farbstoffes entsprechen.

Ich konnte mich nicht mit den oben angeführten Versuchen beschränken; es war notwendig, die Analyse des wie im Glas, so auch auf dem Gewebe erhaitenen Lackes auszuführen.

Bei der. Bereitung, und besonders bei der Reinigung des Lackes, stieb ich auf sebr viele Hindernisse, jedoch gelang es mir, nach zahlreichen Proben und Versuchen lolgendes Rezept auszuarbeiten. Die Tanninlösung wird in der Anwesenheit von Natriumazetat mit der Brechweinsteinlösung präzipitiert, der Niederschlag des antimongerbsauren Salzes auf dem baumwollenen Filter mit Wasser abgespült und in feuchtem Zustande in das Glas mit Wasser hinübergebracht.

In solche Suspensionen des antimongerbsauren Salzes im Wasser wurde die alkoholwässerige Parbstofflossung in einem Quantum hinzugefügt, das die nach der Reaktion berechnete Quantität dreifach oder vierfach übertrifft. Die Mischung wurde im Laufe von 20 Minuten gekocht, der erhaltene Niederschlag des Lackes durch den baumwollenen Filter filtriert, sodann wurde der FarbstoffuberschuB sorgfältig und lange mit heiBem Wasser abgewaschen; der Niederschlag wurde darauf vom Filter in ein Glas gesammelt und mit Alkohol gesotten, filtriert, aufs neue mit Alkohol ausgekocht, wiederum abfiltriert, diese Operation nochmal wiederholt und zuletzt das Präzipitat auf dem Filter mit heißem Alkohot ausgespült, getrocknet und der Analyse auf $\mathrm{Sb}$ und $\mathrm{N}$ unterworfen. - Das beschriebene Verfahren besteht also aus zwei Teilen: aus der Bereitung des antimongerbsauren Salzes und der darauffolgenden Bereitung desselben Lackes. Die Versucike zeigten, dab zum Erhalten eines reinen Lackes ein ganz reines, antimongerbsaures $\mathrm{Sal}_{2}$ notwendig sei, d.h. ein Salz, das kein Tannin oder Brechweinstein in freiem Zustande enthält, da diese letzteren Substanzen, in eine Verbindurig mit dem Farbstoffüberschuß eintretend das gerbsaure Salz des Farbstoffes und wahrscheinlicb eine Verbindung der antimonigen Säure mit dem Farbstoffe geben können, welche zu dem Lack beigemengt werden und weder vom Wasser noch durch den Alkohol entfernt werden können.

Bei der Bereitung des Lackes habe ich absichtlich einen FarbstoffïberschuB angewandt, um zu beweisen, daß nur eine bestimmte Menge des Farbstoffes in eine Reaktion mit dem antimongerbsauren Salze eintritt, und daß der Farbstoffüberschuf durch den sich bildenden Lack nicht adsorbiert wird.

Die Analyse des Stickstoffes in dem Lacke wurde auf übliche Weise nach Dumas geführt, aber es erwies sich die Notwendigkeir, folgende bestimmte Bedingungen bei der Analyse des Antimons festzusetzen. Der bei $100^{\circ} \mathrm{C}$ getrocknete Lack wird in einem bhen Glase durch $5 \ldots . .8 \mathrm{ccm}$ konz. Salpetersäure zersetzt; nachdem die starke Entwicklung dẹr Stickstoffexyde nachgelassen hatte, wurde die Flussigkeit, um die Reaktionsbeendigung zu erreichen, bis zum Sieden erwärmt. Nachdem ich weiter einen gröbteri Teil der Säure durch Abdampten entfernt hatte, fugte ich zur Mischung $30 \mathrm{ccm}$ Wasser und $1-2 \mathrm{ccm}$ konz. Salzsäure hinzu, dampfte die Flüssigkeil anfs neue ab, fügte wiederum siedendes Wasser und die heike Lösung von $2 \mathrm{~g}$ der Weinsäure binzu und filtrierte den durch Oxydieren des organischen Teiles des lackes erhaltenen Niederschlag ${ }^{2}$ ) ab. In das konzentrierte Filtrat wurden noch einmal $5 \mathrm{ccm}$ konz. Salzsäure gegeben und nach diesen Operationen die i.ösung durch Schwefelwasserstoff zuerst in kaitern Zustunde und dann bel der Temperatur von $60-70^{\circ} \mathrm{C}$ präzipitiert. Der Niederschlag von Antimonsulfid wurde turch den Gooch-Tiegel filtriert, mit Wasser und ein wenig Essigsäure ausgewaschen, getrocknet, irn Kohlenstoffdioxydstron bei $280 \cdots 290^{\circ} \mathrm{C}$ geglüht und nach Abikühlen gewogen.

Bei meinen Versuchen bonutze ich folgende Farbstoffe: Chrysoidin $[A]$, Fuchsin diam. krist $[\mathrm{C}]$, Kristallviolett $[\mathrm{B}]$, Brillantgrün $[\mathrm{M}]$, Rodamin extra $[B]$, Viktoriablau $B[M]$ und Safranin $T[B]$. Alle diese Farbstoffe wurden vor der Anwendung kristallisiert.

Ich führe unten die Ziffern der Analysen von zwei zu verschiedener Zeit bereiteten Lacken an.

Chrysoidinlack:

Nr. 1 Einwage $0,4841 \mathrm{~g}$ $0,0860 \mathrm{~g} \mathrm{Sb}_{2} \mathrm{~S}_{3}-0,0614 \mathrm{~g} \mathrm{Sb}$ $=12,69$ Proz. Sb.

, 2 Einwage $0,4581 \mathrm{~g}$ $0,0803 \mathrm{~g} \mathrm{Sb}_{3} \mathrm{~S}_{3} \cdots 0,0574 \mathrm{~g} \mathrm{Sb}$ $=12,52 \mathrm{Proz} . \mathrm{Sb}$.

4) In manchen Fälien war dieser Miederschlag sehr bedeutend und trennte sich sehr leicht von der Flüssigkeit $a b$, zuweilen aber bildete der organische Teil des Lackes beim $\mathrm{Oxydieren} \mathrm{lösliche} \mathrm{Produkte,} \mathrm{welche,} \mathrm{da}$ sie oxydierende Eigenschaften besitzen, beim Präzipitieren durch $\mathrm{H}_{2} \mathrm{~S}$ viel Verdruß verursachten, so das man sie durch Kristallisieren beim Abkühien entfernen mußte. 
Nr. 1 Einwage $0,4812 \mathrm{~g}$ $27,62 \mathrm{ccm} \mathrm{N}$ bei $738 \mathrm{~mm}$ u. $21^{\circ} \mathrm{C}$ $=6,01$ Proz. N.

, 2 , $0,4387 \mathrm{~g}$ $23,05 \mathrm{ccm} \mathrm{N}$ bei $746 \mathrm{~mm} \mathrm{u} .20^{\circ} \mathrm{C}$ $=5,90$ Proz. N.

Nach der Berechnung sind

$\mathrm{Sb}=12,12$ Proz.

$\mathrm{N}=5,66$ Proz. erforderlich.

Fuchsinlack:

Nr. 1 Einwage $0,4216 \mathrm{~g}$ $0,0650 \mathrm{~g} \mathrm{Sb}_{2} \mathrm{~S}_{3}-0,04642 \mathrm{~g} \mathrm{Sb}$ $=11,01$ Proz. Sb.

.2 $\quad 0,04814 \mathrm{~g}$ $0,0771 \mathrm{~g} \mathrm{Sb}_{2} \mathrm{~S}_{3}-0,0551 \mathrm{~g} \mathrm{Sb}$ $=11,44 \mathrm{Proz}$. Sb.

" 1 n $0,5239 \mathrm{~g}$

$18,48 \mathrm{ccm} \mathrm{N}$ bei $750 \mathrm{~mm}$ u. $18^{\circ} \mathrm{C}$ $=4,00$ Proz. $\mathrm{N}$.

" 2 " $0,5121 \mathrm{~g}$

$19,1 \mathrm{ccm} \mathrm{N}$ bei $754 \mathrm{~mm}$ u. $20^{\circ} \mathrm{C}$ $=4,21$ Proz. $\mathrm{N}$

Die theoretisch erforderliche Quantität: $\mathrm{Sb}=11,11$ Proz. $\mathrm{N}=3,88$ Proz.

Kristallviolettiack:

Nr. 1 Einwage $0,5179 \mathrm{~g}$ $0,0740 \mathrm{~g} \mathrm{Sb}_{2} \mathrm{~S}_{3}-0,05285 \mathrm{~g} \mathrm{Sb}$ $=10,2$ Proz. Sb.

.2 , $2,4323 \mathrm{~g}$ $0,0610 \mathrm{~g} \mathrm{Sb}_{2} \mathrm{~S}_{\mathrm{s}}-0,04357 \mathrm{~g} \mathrm{Sb}$

Nr. 1 Einwage $0,4205 \mathrm{~g}$ $=10,01$ Proz. Sb.

$12,20 \mathrm{ccm} \mathrm{N}$ bei $754 \mathrm{~mm}$ u. $16^{\circ} \mathrm{C}$ $=3,34$ Proz. N.

"2 $\quad 0,4699 \mathrm{~g}$

$14,80 \mathrm{ccm} \mathrm{N}$ bei $761 \mathrm{~mm}$ u. $17^{\circ} \mathrm{C}$ $=3,66$ Proz. N.

Die theoretische Berechnung ergibt:

$\mathrm{Sb}=10,43$ Proz. $\mathrm{N}=3,65$ Proz.

Safraninlack:

Nr. 1 Einwage $0,4731 \mathrm{~g}$ $0,0695 \mathrm{~g} \mathrm{Sb}_{2} \mathrm{~S}_{8}-0,0496 \mathrm{~g} \mathrm{Sb}$ $=10,5$ Proz. Sb.

, $2 \quad 0,5113 \mathrm{~g}$ $0,0767 \mathrm{~g} \mathrm{Sb}_{2} \mathrm{~S}_{3}-0,05478 \mathrm{~g} \mathrm{Sb}$ $=10,71$ Proz. Sb.

1 $\quad 0,4023 \mathrm{~g}$

$19,7 \mathrm{~cm} \mathrm{~N}$ bei $730 \mathrm{~mm}$ น. $19^{\circ} \mathrm{C}$ $=5,4$ Proz. $\mathrm{N}$.

.2 $\quad 0,5077 \mathrm{~g}$

$24,1 \mathrm{ccm} \mathrm{N}$ bel $746 \mathrm{~mm} \mathrm{u.} 20^{\circ} \mathrm{C}$ $=5,32$ Proz. N.
Die Berechnung gibt: $S b=10,9$ Proz.

$\mathrm{N}=5,1$ Proz.

Lack aus Viktoriablau:

Nr. $10,4711 \mathrm{~g}-0,0593 \mathrm{~g} \mathrm{Sb}_{2} \mathrm{~S}_{3}-0,04234 \mathrm{~g}$ $\mathrm{Sb}=9,2$ Proz. Sb.

. $20,5221 \mathrm{~g}-0,0681 \mathrm{~g} \mathrm{Sb}_{\mathrm{F}_{2}} \mathrm{~S}_{8}-0,04861 \mathrm{~g}$ $\mathrm{Sb}=9,31$ Proz. Sb.

, $10,4335 \mathrm{~g}-14,4 \mathrm{ccm} \mathrm{N}$ bei $762 \mathrm{~mm}$ $21^{\circ} \mathrm{C}=3.8$ Proz. N.

n $20,5002 \mathrm{~g}-16,46 \mathrm{~cm} \mathrm{~N}$ bei $750 \mathrm{~mm}$ $18^{\circ} \mathrm{C}=3,75$ Proz. N.

nach der Formel aber sind ertorderlich: $\mathrm{Sb}=9,61$ Proz. $\mathrm{N}=3,36$ Proz.

Auf viele Hindernisse stieb ich bei der Analyse der auf dem Gewebe enthaltenen Lacke, da ich für diesen Lack kein Lösungsmittel finden konnte. Es blieb mir also nichts übrig, als zur Analyse des Lackes das ganze Gewebe anzuwenden, was aber nicht so einfach auszuführen war.

Die Arbeitsmethode bestand in folgendem: es wurden mehrere Streifen baumwollenen Gewebes von $5 \mathrm{~cm}$ Breite and $30, \mathrm{~cm}$ Länge bereitet, in Anpullen bei $100^{\circ} \mathrm{C}$ getrocknet und gewogen, nachher wurden sie in eine Tanninlösung $20 \mathrm{~g}$ pro Liter bei $60^{\circ} \mathrm{C}$ bis zum Erkalten der Lösung eingetaucht, darauf herausgenommen, getrocknet und in der Brechweinsteinlösung $(10 \mathrm{~g}$ pro Liter $)$ fixiert. Die sorgfältig, aber vorsichtig gewaschenen Streifen wurden zuerst an der Luft getrocknet und darauf je $z$ wei Streifen mit einem von den oben erwähnten Farbstoffen gefärbt. Die Färbung wurde auf folgende Weise ausgeführt: In ein Porzellangefäb wurde eine kleine Farbstoffmenge eingebracht, in das so hergestellte Bad wurden die Streifen gesenkt, die Temperatur nach und nach bis zu $70-80^{\circ} \mathrm{C}$ gesteigert; nach der Entfärbungsmaligabe des Bades wurden die Farbstoffe aufs neue hinzugefügt; ein solches Verfahren dauerte so lange, bis das Bad völlig erschöpft war.

Eine derartig allmähliche Hinzufügung des Farbstoffes ins Bad ist von großer Wichtigkeit, da bei der Anwendung der konzentrierten Lösungen von Farbstoffen auf der Oberfläche der Pasern sich eine reichliche Ablagerung des Lackes bildete, welche das Durchdringen des Farbstoffes ins Innere der Fasern verhinderte; es blieb also ein gewisser Teil von antimongerbsaurem Salze frei.

Die gefärbten Streifen werden anfangs mit kaltem Wasser abgespült, nachher ins Wasser- 
bad eingetaucht, die Temperatur des Bades bis zu $70-80^{\circ} \mathrm{C}$ gesteigert. Die Streifen blieben im Bade im Laufe von 5-10 Minuten und wurden die ganze Zeit umgerührt. Bei diesen Bedingungen ging vom Gewebe eine gewisse Menge überschüssigen Farbstoffes und ein kleiner Teil vom Lack in die Lösung über; es wurde dabei bemerkt, dak der Lack ein um so geringeres Molekulargewicht hat, je mehr er im Wasser löslich ist, jedoch ist diese Löslichkeit sehr gering.

Die bis zum Erreichen beständigen Gewichts getrockneten und abgewogenen Streifen wurden der Analyse unterworfen, um den Antinon- und Stickstoffbestand zu bestimmen. Die Wägemengen des Lackes wurden aus der Differenz der Gewichte der gefärbten und ungefärbten Streifen ermittelt. Es erwies sich nach meinen Versuchen, daß das Streifgewicht nach verschiedenen Operationen, z. B. nach der Imprägnierung, Abwaschung, Färbung, sich fast nicht verändert, folglich, daß die Differenz von Gewichten der gefärbten und ungefärbten Streifen das Lackgewicht ausdrückt.

Die Antimonbestimmung in dem auf das Gewebe aufgetragenen Lacke wurde auf folgende Weise ausgeführt: Die Streifen wurden in Erlenmeyer'schen Gläsern durch einige ccm konz. Salpetersäure bei schwachem Erwärmen bearbeitet; nach der Zerstörung des baumwollenen Gewebres und des Lackes (was nach der Entfärbung der Lösung bemerkt wurde) wurde zu der Mischung Wasser und Weinsteinsäurelösung zugesetzt, die Lösung vom Niederschlage abfiltriert, das Filtrat bis fast zu völligem Trocknen zur Entfernung der Salpetersäure verdampft, Wasser und Salzsäure (1:3) zugefügt und die Lösung der Wirkung von Schwefelwasserstoff unterworfen. Das Sulfid wurde durch den Goo ch tiegel filtriert und im Strome von Kohlenstoffdioxyd bei $280-290^{\circ} \mathrm{C}$ durchgeglüht und sodann gewogen.

Der Stickstoff wurde nach Kjehldal bestimmt. In runde Kolben $(500 \mathrm{ccm}$ Inhalt) wurden $100 \mathrm{ccm}$ Schwefelsäure eingegossen, die Säure bis auf $200-220^{\circ} \mathrm{C}$ erwärmt und in dieselbe allmählich kleine Stückchen der gefärbten Gewebe hineingelegt. Die Erfahrung zeigte, daß die gleichzeitige Zersetzung durch die Schwefelsäure aller zur Analyse bereiteten gefärbten Streifen höchst unbequem war, da dabei eine rasche Verkohlung anfing und der Oxym dationsproze $B$ sehr langsam verlief. Als $\mathrm{Oxy}$ dationsmittel wurde Kupfervitriol angewandt.
Nachdem die Flüssigkeit in den Kolben durchsichtig geworden war, wurde sie abgekühlt und in jeden Kolben eine Natriumhydratlösung zugesetzt; das ausgetriebene Ammoniak wurde durch ein mit Rückflußkühler versehenes Rohr in eine titrierte Salzsäurelösung geleitet. Nach der Menge der in die Reaktion nicht eingetretenen Säure, welche nach jodometrischer Methode bestimmt wurde, konnte man auf die Stickstoffmenge im Lacke schließen.

Die Analysen verschiedener Lacke werden unten angeführt.

Chrysoidinlack:

$0,5144 \mathrm{~g}-0,0888 \mathrm{~g} \mathrm{Sb}_{2} \mathrm{~S}_{3}-0,06343 \mathrm{~g} \mathrm{Sb}$ $=12,33$ Proz. $0,5040 \mathrm{~g}-$ Gewichtsportion, von der Salzsäure wurden genommen $100 \mathrm{ccm}$, Titer 0,0036852 , im ganzen $0,36852 \mathrm{~g}$, und nach der Analyse blieben $0,28977 \mathrm{~g}$; zur Neutralisation des Ammoniaks wurden $0,07875 \mathrm{~g} \mathrm{HCl}$ verbraucht, was $0,0304 \mathrm{~g} \mathrm{~N}$ oder 6 Proz. entspricht.

Die Berechnung gibt: $\mathrm{Sb}=12,12$ Proz., $\mathrm{N}=5,66$ Proz.

Fuchsinlack:

Einwage $0,4685 \mathrm{~g}-0,0737 \mathrm{~g} \mathrm{Sb}_{2} \mathrm{~S}_{3}$ $-0,0526 \mathrm{~g} \mathrm{Sb}=11,22$ Proz. Sb. Einwage $0,5321 \mathrm{~g}$, von der Salzsäure wurden $50 \mathrm{ccm}$, Titer 0,0036852 , d. h. 0,18426 g genommen; nach der Analyse wurden $0,12564 \mathrm{~g}$ bestimmt, die Neutralisation des Ammoniaks erforderte $0,05862 \mathrm{~g}$, was 4,23 Proz. $\mathrm{N}(0,02251 \mathrm{~g} \mathrm{~N})$ entspricht.

Die berechneten Mengen:

$$
\mathrm{Sb}=11,11 \text { Proz., } \mathrm{N}=3,88 \text { Proz. }
$$

$$
\mathrm{Kristallviolettlack:}
$$

$0,4991 \mathrm{~g}-0,0741 \mathrm{~g} \mathrm{Sb}_{2} \mathrm{~S}_{\mathbf{3}} \ldots 0,05295 \mathrm{~g} \mathrm{Sb}$ $=10,61$ Proz. Sb. Einwage $0,5107 \mathrm{~g}$, von der Säure wurden $50 \mathrm{ccm}$ genommen, 0,0036852 , im ganzen 0,18426 g; nach der Analyse wurden $0,13172 \mathrm{~g}$ bestimmt, die Neutralisation des Ammoniaks erforderte $0,05254 \mathrm{~g}$, was $0,020173 \mathrm{~g}$ N, d. h. 3,95 Proz. N entspricht.

Die berechneten Mengen:

$$
\mathrm{Sb}=10,43 \text { Proz., } \mathrm{N}=3,65 \text { Proz. }
$$

Safraninlack:

$0,5011 \mathrm{~g}-0,0787 \mathrm{~g} \mathrm{Sb}_{2} \mathrm{~S}_{3}-0,0561 \mathrm{~g} \mathrm{Sb}$ $=11,2$. Proz. Sb. Einwage $0,5273 \mathrm{~g}$, von der Säure wurden $50 \mathrm{ccm}$, des Tit. 0,0036825 , im ganzen $0,184125 \mathrm{~g}$; nach der Analyse wurden $0,109695 \mathrm{~g}$ bestimmt, die Neutralisation des Ammoniaks erforderte $0,07443 \mathrm{~g}$, was $0,02858 \mathrm{~g}$ N., d. h. 5,42 Proz. N entspricht. 
Der Formel entspricht: $\mathrm{Sb}=10,9$ Proz:, $N==5,1$ Prox.

Beim Vergleich der berechneten und der als Resultat der Beobachtung erhaltenen Zahlen kann man sehen, dafs diese in genügender Weise im Einklange stehen. Es unterliegt also keinem $Z$ weifel, taß bei Zusammenwirkung zwischen dem antimongerbsauren Salze und dem Farbstoffe derselbe in eine chemische Verbindung mit dem Antimon eintritt und dabei die Salzsüure frei wird.
Zum Schlub soll nochmals erwähnt werden, dah im allgemeinen Palle, obne die Erfülung bestinmter Versuchsbedingungen, das Tannin, der Brechweinstein und der basische Farbstroff bei gegenseitiger Reaktion Adsorptionsverbindingen bilden, und dab nur in speziellen Fällen, wie eben bei der technischen Färbung der Baumwollfasern mit basischen Farbstoffen, stöchio. metrische Verbindunger erhalten werden können,

Kiew, September 1913.

\title{
Organosole von Metallen der Platingruppe, I.
}

\author{
Von Conrad Amberger.
}

(Mltieilung aus der Köningl. Untersuchungsanstalt Erlangen.)

(Eing. 13. Mărz 1913.)

In zwei Miteilungen it wurde gezeigt, daß es in Cegerwart von Wollfett oder dessen unverseifbaren Anteiten - - den Wollett-Alkoholen als Schutzkolioide gelingt, die Metalle Siber und Gold in kolloide Formen überzuführen, welche die bemerkenswerte Eigenschaft besitzen, mit allen den Lösungsmilteln, die Wollfett lösen, Organosole zu bilden. Im nachstehenden soll uber die Darstellung der Organosole der Platingruppe berichter werden.

Das Wollfett besitzt bekanntlich die Eigenschaf, in hohem MaRe Wasser oder wässerige Losumgen in sich autzunehmen, ohne dab dabei die salbenartige Kunsistenz des Wollfeltes geändert wird. Verreibt man nun mit wässerigen Lösungen von Salzen des zweiwertigen Platins oder Palladiums imprägivertes Wollfett mit atzenden Alkalien oder Alkalikarbonaten, so eatstehen durch doppelte Umsetzung die Hydroxyde des Platins bzw. Palladiums. Erwärmt man diese Zwischenprodukte, so tritt erst nach längerem Erwärmen eine von Dunkelfärbung begleitete, geringfügige Reduktion ein. Die reduzierende Wirkung gewisser im . Wollfett vorhandener Bestandteile erweist sich im Vergleiche nit den entsprechenden Unsetzungsprodukten des Silbers (1. c.) den Verbindungen der Platinmetalle gegenutber als zu schwach. Es mußte daher nocls ein Reduktionsmittel zugegeben werden, als welches sich Hydracinhydrat besonders eignet, während Formaldehyd and Hydroxylamin, die ebenfalls versucht wurden, keme betiedigend: Resultate gaben. Die nach dicsem Verabren ehatener kolloiden Platinmetalle lassen sich aus ihren lösungen in Petrolather oder Chloroform mit organischen

5) Koll. - Zeitscht. 11, 97-102 (1912). .
Lösungsmitteln, die Wollfett wenig oder gar nicht lösen, fällen. Diese Niederschläge lossen sich, wenn die Fallung unter geeigneten Konzen. trationsbedingungen vorgenommen wird, wieder in Wollfett lösenden Flüssigkeiten als Organosole. Besonders geeignet als Fallungsmittel erwies sich Alk ohol, durch den das Metall als testes Organosol vollständig gefällt wird, während ein Teil des Wollfetes in Lösung bleibt. Es labt sich also durch dieses Verfahren eine Anreicherung der Präparate an dem metallischen Organosol erzielen.

\section{Kolloides Palladium.}

Sehr verdünnte, kolloide Palladiumlösungen sind von A. Lottermoser ${ }^{2}$ ) durch Reduktion von Palladiochlorid mit Formaldehyd und ron G. Bredigs) durch elektrische Kathodenzerstäibang unter Wasser dargestellt worden. Hochprozentige Praparate von lrolloidem Palladiun sind in Kombination mit protalbinsaurem Natrium von C.Paal und C. Amberger ${ }^{\text {a) }}$ in festem Zustande erhalten worden; unter Verwencung von Gummi als Schutzkolloid gelangten A. Gutbier and $G$. Hof meier $\mathrm{r}^{5}$ zu kolloidem Palladiun, dab sich jedoch auf diesem Wege nicht in fester, beständiger form gewinnen lie\&.

Darstellugen von Palladiumorganosolen unter Verwendung von Wollfett als Scbutzkolloid.

$1.7 \mathrm{~g}$ Palladiunchorü $(=1$ Teil Pailadium) wurden in einer Mischung von 1 Tei rauchende!

2) Ueber anorg. Kolloide (Stuttgart 1904), 34

8) Anorg, Fermente (Leipzig 1901) 30.

4) Ber, d. Deutsch. chem. Ges. 37, 132-139 (1904).

5) Journ. prakt. Chem. (2), 71, 358-365 (1905). 